\title{
Delta-9-tetrahydrocannabinol increases striatal glutamate levels in healthy individuals: implications for psychosis
}

\author{
Marco Colizzi $\mathbb{1}^{1} \cdot$ Nathalie Weltens ${ }^{2} \cdot$ Philip McGuire ${ }^{1} \cdot$ David Lythgoe $^{3} \cdot$ Steve Williams $\mathbb{1}^{3} \cdot$ \\ Lukas Van Oudenhove ${ }^{2} \cdot$ Sagnik Bhattacharyya $\mathbb{1}^{1}$
}

Received: 21 May 2018 / Revised: 6 December 2018 / Accepted: 26 December 2018 / Published online: 15 February 2019

(c) The Author(s) 2019. This article is published with open access

\begin{abstract}
The neurobiological mechanisms underlying the association between cannabis use and acute or long-lasting psychosis are not completely understood. While some evidence suggests altered striatal dopamine may underlie the association, direct evidence that cannabis use affects either acute or chronic striatal dopamine is inconclusive. In contrast, pre-clinical research suggests that cannabis may affect dopamine via modulation of glutamate signaling. A double-blind, randomized, placebo-controlled, crossover design was used to investigate whether altered striatal glutamate, as measured using proton magnetic resonance spectroscopy, underlies the acute psychotomimetic effects of intravenously administered delta-9-tetrahydrocannabinol $(\Delta 9$-THC; $1.19 \mathrm{mg} / 2 \mathrm{ml}$ ), the key psychoactive ingredient in cannabis, in a set of 16 healthy participants (7 males) with modest previous cannabis exposure. Compared to placebo, acute administration of $\Delta 9$-THC significantly increased Glutamate (Glu) + Glutamine (Gln) metabolites (Glx) in the left caudate head $(P=0.027)$. Furthermore, compared to individuals who were not sensitive to the psychotomimetic effects of $\triangle 9$-THC, individuals who developed transient psychotic-like symptoms ( $\sim 70 \%$ of the sample) had significantly lower baseline Glx (placebo; $P 7=0.023$ ) and a 2.27-times higher increase following $\triangle 9$-THC administration. Lower baseline Glx values $(r=-0.55 ; P=0.026)$ and higher previous cannabis exposure $(r=0.52 ; P=0.040)$ were associated with a higher $\triangle 9$-THC-induced Glx increase. These results suggest that an increase in striatal glutamate levels may underlie acute cannabis-induced psychosis while lower baseline levels may be a marker of greater sensitivity to its acute psychotomimetic effects and may have important public health implications.
\end{abstract}

Supplementary information The online version of this article (https:// doi.org/10.1038/s41380-019-0374-8) contains supplementary material, which is available to authorized users.

Sagnik Bhattacharyya

sagnik.2.bhattacharyya@kcl.ac.uk

1 National Institute for Health Research (NIHR) Biomedical Research Centre (BRC), South London and Maudsley NHS Foundation Trust, and Department of Psychosis Studies, Institute of Psychiatry, Psychology and Neuroscience, King's College London, London SE5 8AF, UK

2 Laboratory for Brain-Gut Axis Studies (LaBGAS), Translational Research Center for Gastrointestinal Disorders (TARGID), Department of Chronic Diseases, Metabolism and Ageing, University of Leuven, Leuven 3000, Belgium

3 Department of Neuroimaging, Institute of Psychiatry, Psychology and Neuroscience, King's College London, London SE5 8AF, UK

\section{Introduction}

Cannabis is the most widely used illicit drug in Europe and over the world, with approximately 200 million users [1] and an estimated 13 million individuals with cannabis dependence [2]. It represents a public health concern as cannabis use can induce transient psychotic symptoms [3-5] and trigger the onset of psychosis in vulnerable individuals [6]. Moreover, cannabis use can exacerbate psychotic symptoms [7-9] and increase the risk of relapse [10-12] in patients with established psychosis in a dosedependent manner [13].

Cannabis exerts its psychotomimetic effects primarily through its psychoactive component delta-9tetrahydrocannabinol $(\Delta 9-\mathrm{THC})[14-16] . \Delta 9-\mathrm{THC}$ is a partial agonist at the endocannabinoid receptor type 1 (CB1), which is widely expressed throughout the brain [17] and downregulated in response to sustained cannabis use [18]. $\triangle 9$-THC has consistently been shown to stimulate the neuronal firing of mesolimbic dopamine neurons 
and elevate striatal dopamine levels in animal models [19]. However, acute administration of $\Delta 9$-THC has been shown to induce striatal dopamine release in some [20-22] but not all human studies [23, 24] (also reviewed in [25]), while a deficit in striatal dopamine release has been reported in cannabis dependence [26]. Additional evidence suggests that $\triangle 9$-THC disrupts striatal function [27], and genetic variation in dopamine signaling modulates this effect [16].

The difficulty in capturing the acute effect of $\triangle 9$-THC on striatal dopamine in man may be explained by the biological distance between $\triangle 9$-THC effects and dopamine dysregulation, as evidence suggests that $\Delta 9$-THC does not affect dopamine release directly but via CB1-dependent modulation of glutamate signaling [17]. Converging evidence from preclinical studies indicates that acute $\Delta 9$-THC administration induces a dose-dependent increase in cortical extracellular, striatal, and hippocampal intracellular glutamate levels through the activation of CB1 receptors at glutamatergic presynapses in cortical and subcortical brain regions, reflecting a reduction in synaptic glutamate levels and receptor functioning [28-30], also reviewed here [31]. A limited number of studies consistently support the evidence for altered brain glutamate levels as measured by proton magnetic resonance spectroscopy (1H-MRS) in otherwise healthy chronic cannabis users, with all [32-35] but one [36] of the five studies indicating reduced levels of glutamate-derived metabolites Glutamate (Glu) or Glutamate + Glutamine $(\mathrm{Glx})$ in both cortical and subcortical brain areas. The only study not showing an effect of cannabis on glutamate in man investigated a modestly sized sample of cannabis users with concurrent methamphetamine use [36]. In contrast, another study conducted in a larger sample suggested reduced Glx metabolite concentration also in individuals with a history of other illicit drug use [37]. However, the cross-sectional case-control design of these studies does not allow one to infer a cause-effect relationship underlying the observed association between cannabis use and glutamatergic alterations in the brain.

To our knowledge, no study has as yet investigated the acute effect of $\Delta 9$-THC on brain glutamate levels in man as a potential mechanism underlying its psychotomimetic effects. Therefore, we employed a placebo-controlled acute pharmacological challenge design to investigate the acute effect of $\Delta 9$-THC administration on brain glutamate levels in man. We focused on three brain regions, the striatum, the hippocampus, and the anterior cingulate cortex (ACC), as preclinical studies suggested that acute $\triangle 9$-THC administration increased glutamate levels not only in the striatum but also in other brain regions, such as the prefrontal cortex and hippocampus [28-30].

Evidence suggests that (1) $\Delta 9$-THC administration in animal models increases glutamate levels in the striatum
[30], (2) $\Delta 9$-THC-induced increase in glutamate levels leads to an excess striatal dopamine via neuronal circuitry involving hypofunctioning N-methyl-D-aspartate (NMDA) receptors [28], and (3) $\Delta 9$-THC-induced modulation of striatal activation is related to the severity of acute psychotomimetic effects induced by it in humans [15, 16, 27]. Hence, we specifically hypothesized that (1) acute $\Delta 9$-THC administration would be associated with an increase in striatal glutamate-derived metabolites; (2) $\Delta 9$-THC-induced striatal glutamate increase would be associated with the development of psychotomimetic symptoms. Based on the limited evidence of a blunted effect of acute $\Delta 9$-THC administration on neurochemical markers (brain-derived neurotrophic factor (BDNF)) in cannabis users compared to healthy subjects [38], the following hypothesis was also tested: (3) previous cannabis exposure would modulate the acute effect of $\Delta 9$-THC on striatal glutamate. We also carried out exploratory analyses to examine whether the acute effects of $\triangle 9$-THC on brain glutamate levels in man were specific to the striatum or also noted in the hippocampus and ACC.

\section{Methods}

A detailed description of the experimental procedure, psychopathological assessment, image acquisition, 1H-MRS quantification, and statistical analyses is provided in Supplementary Methods and is summarized here briefly.

We employed a double-blind, randomized, placebocontrolled, crossover design, with counterbalanced order of drug administration, using an established protocol [16, 39]. Sixteen right-handed healthy participants ( 7 males), abstinent from cannabis for at least 6 months and with no history of alcohol abuse, nicotine dependence, or illicit drug use, were assessed on two different occasions separated by at least a 2-week interval, with each session preceded by intravenous administration of $\Delta 9-\mathrm{THC}(1.19 \mathrm{mg} / 2 \mathrm{ml})$ or saline. A power analysis indicated that a total sample of 16 people would allow detection of a medium effect $(d=0.65)$ with $80 \%$ power using a one-tailed paired $t$-test.

Immediately before and at $20 \mathrm{~min}$ and $2.5 \mathrm{~h}$ after drug administration, psychopathological ratings [40-43] were recorded by an expert clinical researcher.

1H-MRS spectra (Point RESolved SpectroscopyPRESS; TE $=30 \mathrm{~ms} ; \mathrm{TR}=3000 \mathrm{~ms} ; 96$ averages) were acquired on a 3 Tesla MR system in the left caudate head, ACC, and hippocampus (Fig. 1), employing the standard GE probe (proton brain examination) sequence, which uses a standardized chemically selective suppression (CHESS) water suppression routine [44] that has been employed before at this centre [45-47]. Data were analyzed with LCModel version 6.3-1L [48]. 
a

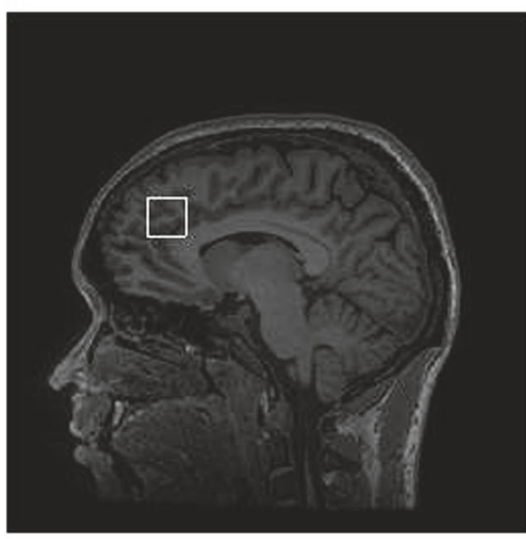

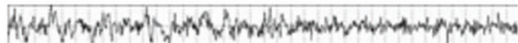

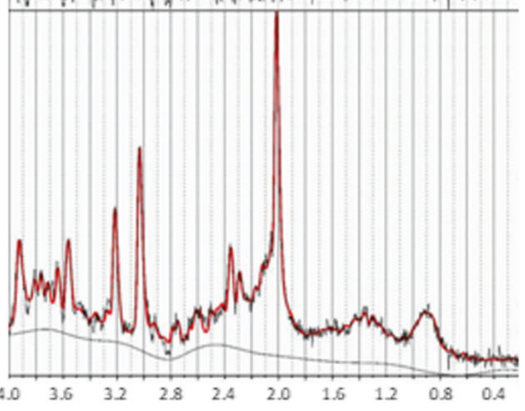

b
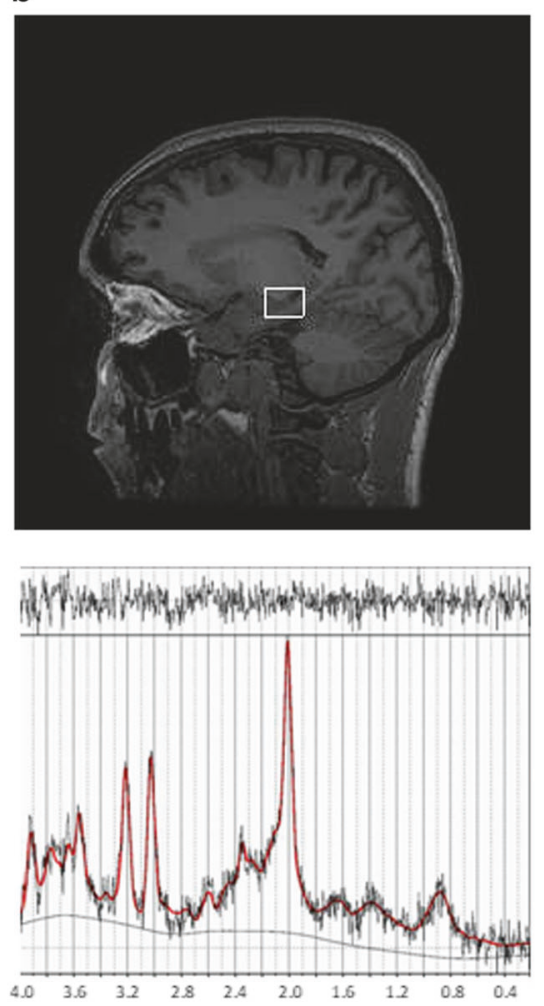

C
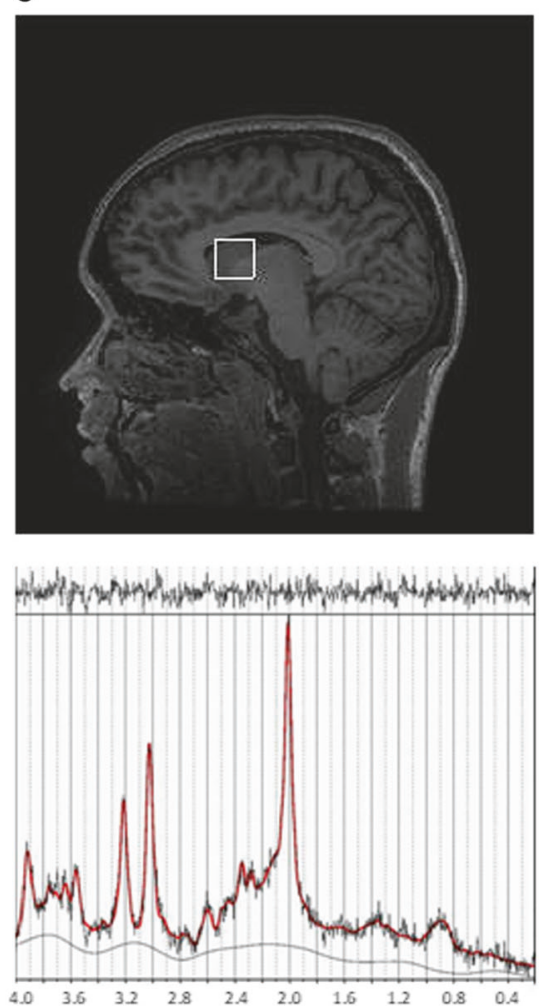

Fig. 1 1H-MRS data acquisition. 1H-MRS, proton magnetic resonance spectroscopy. a Left anterior cingulate cortex (ACC), b left hippocampus, and $\mathbf{c}$ left head of the caudate

Data was normally distributed. Paired $t$-tests were used to estimate the effects of $\Delta 9$-THC on symptoms (focusing on peak changes from the baseline, as we did not expect symptom manifestation by the 2.5 -h time point [14]) and striatal Glx values, and $t$-tests to compare $\Delta 9$-THC-induced Glx changes between subjects sensitive to and those not sensitive to the psychotomimetic effects of $\triangle 9$-THC, based on the manifestation of clearly detectable primary symptoms of psychosis ( $\geq 2$-point increase in Positive and Negative Syndrome Scale (PANSS) [40] delusions, hallucinations, unusual thought content, suspiciousness, and grandiosity items), as drawn from previous factor analytic work [49] as well as previous work to characterize acute sensitivity to $\triangle 9$-THC [50]. Pearson correlation analyses were used to test for an association between changes in striatal Glx values and previous cannabis exposure (SPSS version 22; SPSS Inc., Chicago, IL).

The composite Glx peak has been widely used as a marker of glutamatergic function, because it likely predominantly reflects glutamate levels, which are typically 5-6 times higher than those of glutamine [51]. Many of the functions of glutamine are connected to the formation of glutamate and the glutamate/glutamine cycle has to be seen as a bi-directional cycle involved in key aspects of metabolism and synaptic function [52]. Research evidence suggests a close coupling of overall neuronal activity and glutamate-glutamine fluxes, with cortical synaptic glutamate release and glutamate-glutamine cycling consuming approximately $60-80 \%$ of the energy produced by oxidative metabolism of glucose. This evidence suggests that synaptic glutamate-glutamine cycling cannot be differentiated from overall glutamate metabolism [53]. Therefore, Glx, the main outcome measure of the MRS study presented here, reflects the total glutamatergic pool available for synaptic/metabolic activity [54].

\section{Results}

\section{Demographic variables, physiological measures, and whole-blood $\triangle 9$-THC levels}

Study participants had a mean age of 24.44 years (SD: 4.29). They had a mean of 16.94 (SD: 2.84) years of education.

Placebo administration had no effect on systolic $(\mathrm{mmHg}$, $M \pm \mathrm{SD}$; baseline: $117.31 \pm 13.26$; drug: $118.56 \pm 9.75 ; P>$ 0.1 ) and diastolic blood pressure (baseline: $62.5 \pm 9.32$; 

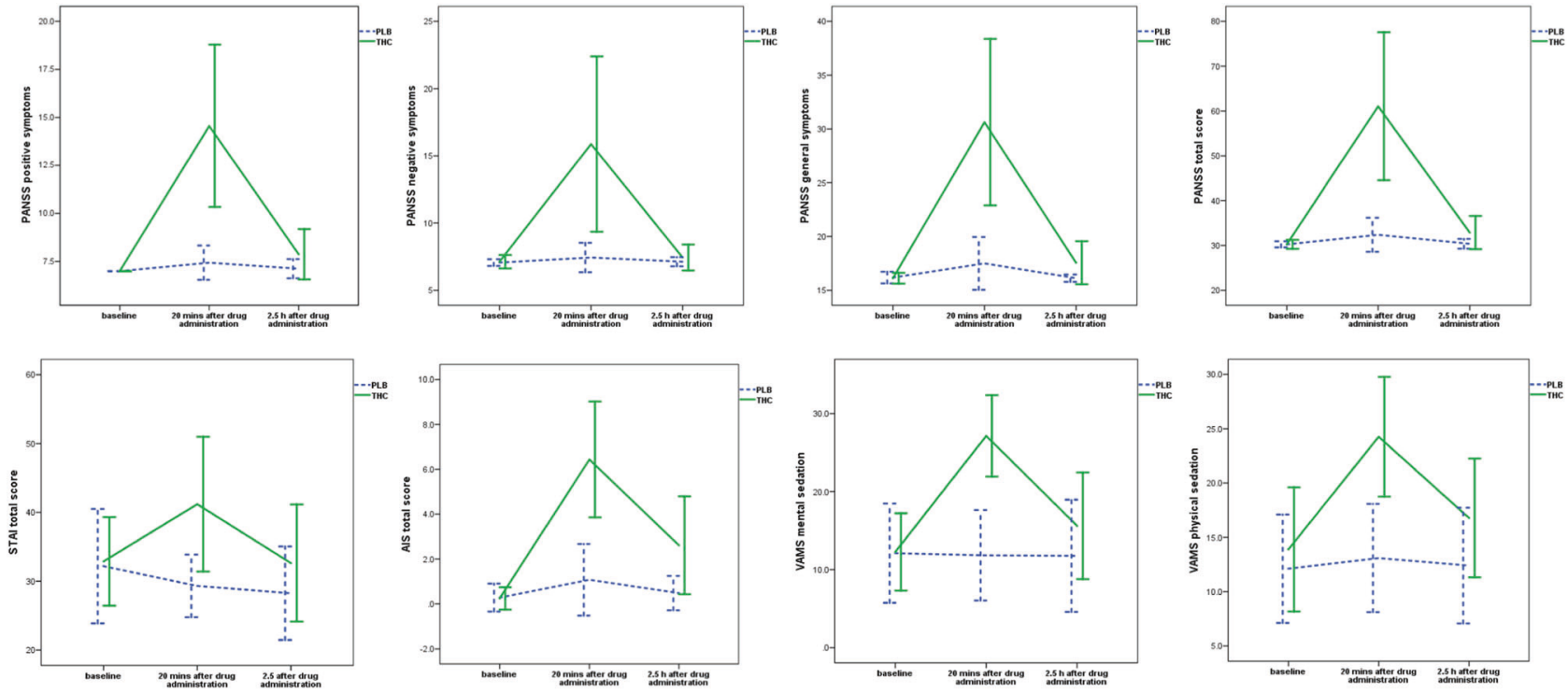

Fig. 2 Acute effect of $\Delta 9$-THC on psychopathological measures. THC (-)-trans- $\Delta^{9}$-tetrahydrocannabinol, PLB placebo, PANSS Positive and Negative Syndrome Scale, STAI State-Trait Anxiety Inventory, AIS

Analog Intoxication Scale, VAMS Visual Analog Mood Scale, mins minutes, h hours, $P$ 2-tailed; error bars show standard deviations

drug: $66.38 \pm 10.56 ; P>0.05$ ), and heart rate (beats per minute; baseline: $69.44 \pm 13.28$; drug: $70.94 \pm 13.90, P>$ $0.1)$. $\Delta 9$-THC administration had no effect on systolic (baseline: $117.13 \pm 10.35$; drug: $117.81 \pm 11.78 ; P>0.1$ ) and diastolic blood pressure (baseline: $64.25 \pm 9.28$; drug: $66.13 \pm 7.05 ; P>0.1)$ but a significant effect on heart rate (baseline: $68.69 \pm 12.72$; drug: $89.31 \pm 22.57, t=4.65$, $P<0.001)$.

The $\Delta 9$-THC plasma levels ( $M \pm \mathrm{SE}$; gas chromatographymass spectrometry, GC-MS) reached a peak $20 \mathrm{~min}$ after drug administration $(220.2 \pm 34.1 \mathrm{ng} / \mathrm{mL})$, and then began to fall ( $2.5 \mathrm{~h}$ after drug administration: $54.6 \pm 8.6 \mathrm{ng} / \mathrm{mL}$ ).

\section{Acute effect of $\triangle 9$-THC on psychopathological measures}

As expected, administration of $\Delta 9-\mathrm{THC}$ was associated with acute induction of transient psychotic symptoms (PANSS positive symptoms subscale, $t=6.62, P<0.001$; PANSS negative symptoms subscale, $t=4.95, P<0.001$; PANSS general symptoms subscale, $t=6.85, P<0.001$; PANSS total score, $t=6.77, P<0.001)$. Also, $\Delta 9$-THC induced an acute and transient increase in symptoms of anxiety (STAI scale, $t=3.72, P=0.002$ ). Finally, subjects experienced significant $\Delta 9$-THC-induced intoxication (AIS, $t=9.41, P<0.001)$ and sedation (VAMS mental sedation subscale, $t=7.72, P<0.001$; VAMS physical sedation subscale, $t=4.90, P<0.001$; Fig. 2). Eleven subjects (69\%) were identified as sensitive to the psychotomimetic effects of $\triangle 9$-THC as determined on the basis of $\geq 2$-point increase in the relevant PANSS items (as described in Methods)

[49]. They had a $5.91( \pm 4.18)$ point increase in the primary symptoms of psychosis compared to a 0.6 point increase $( \pm 0.55)$ for the remaining subjects (drug effect, $t=4.13$, $P=0.002)$.

\section{H-MRS results}

Voxel segmentation and spectral quality are reported in Table 1.

\section{Striatal Glx measures}

As hypothesized, acute $\triangle 9$-THC administration increased Glutamate (Glu) + Glutamine (Gln) metabolites (Glx) in the left caudate head (placebo: $10.03 \pm 2.25$; $\Delta 9$-THC: $12.22 \pm$ $3.49 ; t=2.09, P=0.027$; effect size: 0.75; Fig. 3). There was an inverse relationship between baseline Glx values (as measured under the placebo condition) and change in Glx induced by acute $\triangle 9$-THC administration. This was such that, the lower the Glx values under placebo, the higher was the increase following $\triangle 9$-THC administration $(r=-0.55$; $P=0.026$ ). Furthermore, there was a positive correlation between previous cannabis exposure (as indexed using lifetime number of times of cannabis use) and $\Delta 9$-THCinduced increase in Glx (drug effect, $r=0.52 ; P=0.040$ ).

Glx values under the placebo condition were significantly lower in subjects who were sensitive to $\triangle 9$-THCinduced psychotomimetic effects $(9.20 \pm 1.93)$ compared to subjects who were not $(11.85 \pm 1.93 ; t=2.54, P=0.023)$. Following acute $\triangle 9$-THC administration, compared to subjects who were not sensitive to the psychotomimetic 
Table 1 Voxel segmentation and spectral quality

\begin{tabular}{|c|c|c|c|c|}
\hline \multirow{2}{*}{$\begin{array}{l}\text { Brain region } \\
\text { Parameter }\end{array}$} & \multirow{2}{*}{$\begin{array}{l}\Delta 9-\mathrm{THC} \\
M(\mathrm{SD})\end{array}$} & \multirow{2}{*}{$\begin{array}{l}\text { PLB } \\
M(\mathrm{SD})\end{array}$} & \multicolumn{2}{|c|}{ Statistics } \\
\hline & & & $t$ & $P$ va \\
\hline \multicolumn{5}{|l|}{ Left caudate head } \\
\hline \multicolumn{5}{|l|}{ Cramér-Rao lower bound } \\
\hline Glu & $9.25(2.35)$ & $9.00(1.83)$ & 0.37 & 0.71 \\
\hline Glx & $11.12(3.54)$ & $10.87(2.58)$ & 0.24 & 0.81 \\
\hline$N A A+N A A G$ & $3.62(1.15)$ & $3.19(0.54)$ & 1.52 & 0.15 \\
\hline $\mathrm{Cr}$ & $3.37(0.62)$ & $3.50(0.89)$ & -0.56 & 0.58 \\
\hline$m I$ & $8.07(3.47)$ & $9.67(4.67)$ & -1.50 & 0.15 \\
\hline$G P C+P C h$ & $4.31(0.79)$ & $4.44(1.46)$ & -0.34 & 0.74 \\
\hline Full width at half maximum & $0.07(0.01)$ & $0.06(0.01)$ & 1.91 & 0.08 \\
\hline Signal to noise ratio & $16.50(3.92)$ & $17.88(3.69)$ & -1.14 & 0.27 \\
\hline Grey matter (\%) & $48.77(7.05)$ & $49.32(5.65)$ & -0.28 & 0.78 \\
\hline White matter (\%) & $49.40(7.26)$ & $48.84(6.73)$ & 0.31 & 0.76 \\
\hline Cerebrospinal fluid (\%) & $1.81(1.86)$ & $1.80(1.79)$ & 0.02 & 0.99 \\
\hline \multicolumn{5}{|l|}{ Left anterior cingulate cortex } \\
\hline \multicolumn{5}{|l|}{ Cramér-Rao lower bound } \\
\hline Glu & $5.69(0.79)$ & $5.94(1.06)$ & -0.77 & 0.45 \\
\hline Glx & $6.31(0.87)$ & $6.75(1.06)$ & -1.81 & 0.09 \\
\hline$N A A+N A A G$ & $2.69(0.48)$ & $2.69(0.60)$ & 0.00 & 1.00 \\
\hline $\mathrm{Cr}$ & $2.75(0.45)$ & $2.62(0.62)$ & 0.70 & 0.50 \\
\hline$m I$ & $4.69(0.60)$ & $5.12(1.89)$ & -0.92 & 0.37 \\
\hline$G P C+P C h$ & $3.25(0.58)$ & $3.25(0.45)$ & 0.00 & 1.00 \\
\hline Full width at half maximum & $0.04(0.01)$ & $0.03(0.01)$ & 1.78 & 0.10 \\
\hline Signal to noise ratio & $24.56(5.20)$ & $25.44(5.50)$ & -0.61 & 0.55 \\
\hline Grey matter $(\%)$ & $67.64(4.43)$ & $67.06(5.14)$ & 0.94 & 0.36 \\
\hline White matter (\%) & $10.94(2.59)$ & $11.85(2.46)$ & -1.59 & 0.13 \\
\hline Cerebrospinal fluid (\%) & $21.26(5.36)$ & $20.94(5.36)$ & 0.45 & 0.66 \\
\hline \multicolumn{5}{|l|}{ Left hippocampus } \\
\hline \multicolumn{5}{|l|}{ Cramér-Rao lower bound } \\
\hline Glu & $9.87(1.82)$ & $9.94(2.52)$ & -0.10 & 0.92 \\
\hline Glx & $9.50(2.85)$ & $10.31(2.73)$ & -0.90 & 0.38 \\
\hline$N A A+N A A G$ & $4.00(1.21)$ & $4.75(1.13)$ & -2.16 & 0.05 \\
\hline $\mathrm{Cr}$ & $4.06(0.68)$ & $4.19(0.75)$ & -0.81 & 0.43 \\
\hline$m I$ & $5.37(1.09)$ & $6.06(1.65)$ & -2.11 & 0.05 \\
\hline$G P C+P C h$ & $4.06(0.44)$ & $4.19(0.83)$ & -0.62 & 0.54 \\
\hline Full width at half maximum & $0.07(0.01)$ & $0.07(0.01)$ & 0.95 & 0.36 \\
\hline Signal to noise ratio & $12.81(2.14)$ & $12.13(2.28)$ & 1.14 & 0.27 \\
\hline Grey matter $(\%)$ & $59.86(8.12)$ & $60.23(6.58)$ & -0.20 & 0.85 \\
\hline White matter (\%) & $36.42(8.96)$ & $35.68(7.60)$ & 0.36 & 0.72 \\
\hline Cerebrospinal fluid (\%) & $3.69(1.39)$ & $4.06(1.56)$ & -1.39 & 0.19 \\
\hline
\end{tabular}

$\triangle 9$-THC delta-9-tetrahydrocannabinol, $P L B$ placebo, Glu glutamate, Glx glutamate + glutamine, $N A A+N A A G \mathrm{~N}$-acetylaspartate $+\mathrm{N}$-acetylaspartylglutamate, $\mathrm{Cr}$ creatine, $\mathrm{mI}$ myo-inositol, $\mathrm{GPC}+\mathrm{PCh}$ Glycerophosphocholine + phosphocholine

effects $(13.01 \pm 3.02)$, subjects sensitive to the psychotomimetic effects of $\Delta 9$-THC had a 2.27-times higher increase in Glx values $(11.85 \pm 3.76)$. However, this difference failed to reach significance $(P>0.1 ;$ Fig. 4$)$.

Acute 49 -THC administration had no effect on ACC (placebo: $19.47 \pm 3.04 ; \Delta 9$-THC: $20.11 \pm 2.04$ ) and hippocampal Glx (placebo: $11.74 \pm 2.32 ; \Delta 9$-THC: $12.29 \pm 2.30$; all $P>0.1$ ). Other metabolite levels are reported in Supplementary Table 1 .

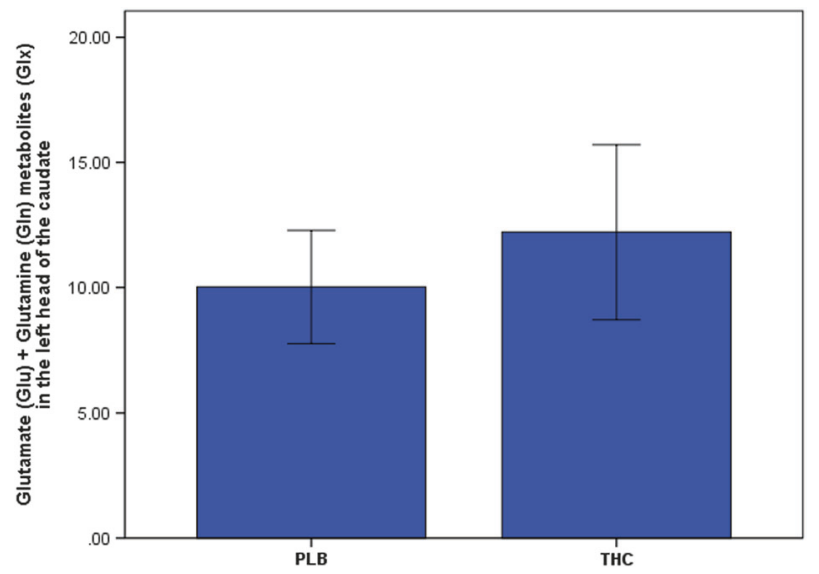

Fig. 3 Acute effect of $\Delta 9$-THC on glutamate measures in the left head of the caudate. THC (-)-trans- $\Delta^{9}$-tetrahydrocannabinol, PLB placebo, $P$ 1-tailed; error bars show standard deviations

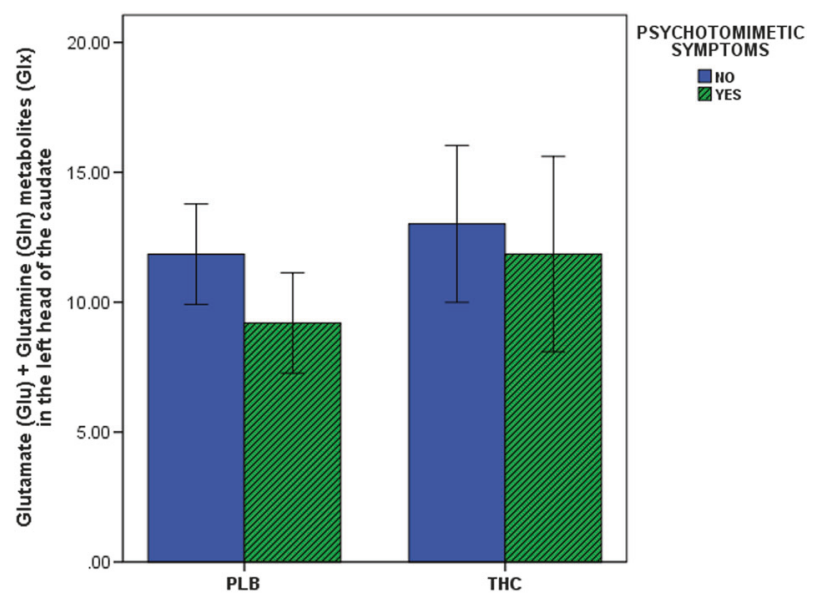

Fig. 4 Acute effect of $\Delta 9$-THC on glutamate measures in the left head of the caudate as a function of the psychotomimetic symptom manifestation. THC (-)-trans- $\Delta^{9}$-tetrahydrocannabinol, PLB placebo, $P$ 2-tailed; error bars show standard deviations

\section{Discussion}

This is the first human study to investigate the acute effect of intravenous $\Delta 9$-THC administration on brain glutamate levels, and whether glutamate level alterations underlie the acute psychotomimetic effects of $\Delta 9$-THC. Consistent with our first hypothesis and with previous evidence from animal studies [28-31] we found that acute administration of $\Delta 9$ THC significantly increased Glx levels in the left caudate head compared to the placebo. As predicted, we also found that this was associated with transient psychotomimetic effects induced by $\Delta 9$-THC. Our most novel finding is that individuals who experienced transient psychotomimetic effects following $\Delta$ 9-THC ( $70 \%$ of the sample) had significantly lower baseline Glx (as under placebo) and an almost two-and-a-half-fold higher increase in Glx following $\Delta$ 9-THC administration compared to individuals who were 
not sensitive to the psychotomimetic effects of $\Delta 9$-THC. Finally, consistent with our prediction, previous cannabis exposure was positively associated with $\Delta 9$-THC-induced Glx increase. Exploratory analyses also suggested that the acute effects of $\triangle 9$-THC on human brain Glx levels are region-specific, as $\Delta 9-\mathrm{THC}$ administration increased Glx levels in the striatum, but not in the hippocampus or the ACC.

In addition to the brain stem projections, the key inputs to the striatum are mesolimbic dopaminergic (DA) projections from the ventral tegmental area (VTA) as well as cortical and thalamic glutamatergic projections [55, 56]. The activity of VTA DA neurons in vivo is dominated by pacemaker-like tonic firing interrupted by phasic bursts leading to striatal dopamine release [57], and this shift from tonic pacemaker firing to bursting is strongly controlled by synaptic input from glutamatergic and GABAergic afferents to the dopamine neurons [58]. Upon acute exposure, endocannabinoids regulate synaptic strength by acting on glutamatergic afferents to VTA dopamine neurons via activation of CB1 receptors [59]. Preclinical evidence also indicates that the intravenous administration of $\Delta 9$-THC can increase VTA dopamine neuronal activity, being ultimately responsible for an increase in striatal dopamine levels through the mesolimbic pathway [19]. However, in vitro studies have demonstrated that cannabinoids do not affect dopamine concentrations when locally applied in the striatum [60], while increasing dopaminergic firing when administered in the VTA [61]. Since VTA dopamine neurons do not express CB1 receptor protein nor mRNA [62], this argues against a direct effect of cannabinoids on dopamine neuron activity, also potentially accounting for the inconsistent evidence on effects of $\Delta 9$-THC administration on striatal dopamine release in man [20-25]. In contrast, the glutamatergic inputs to the striatum are especially relevant as they are involved in the processing of different stimuli, such as rewarding and stressful information, and the selection of related behavioral responses [63]. In line with this, acute $\Delta 9$-THC administration in animal models has been shown to consistently increase glutamate levels in a number of brain regions including the striatum [28-31]. Therefore, glutamate rather than dopamine may play a more important role in the neurochemical underpinnings of the acute psychotomimetic effects of cannabis [31]. However, no acute challenge study had investigated the effect of acute administration of $\Delta 9$-THC on glutamate metabolism in humans. In sum, our study confirms preclinical evidence [28-31] that a single dose of $\Delta 9$-THC may increase striatal glutamate levels and suggests this as a potential mechanism underlying the acute psychotomimetic effects of cannabis.

Previous preclinical research indicates that a history of psychostimulant self-administration leads to decreased basal glutamate in both the striatum and the primary neuronal source of striatal glutamate, the prefrontal cortex. Instead, acute psychostimulant drug administration in abstinent animal models previously exposed to the drug induces an enhancement of cortical and striatal glutamate release not seen in drug-naive subjects [64, 65]. Drug-induced heightened activation of cortical glutamatergic afferents to the VTA has been proposed to modulate behavioral sensitization and addiction [66]. In line with this evidence, we found that the lower the striatal Glx levels at baseline, the higher was the increase after $\Delta 9$-THC administration. Furthermore, we found that the higher the previous cannabis exposure, the higher was the $\Delta 9$-THC-induced striatal increase, potentially suggesting sensitization due to the effects of previous cannabis exposure. However, an important caveat to such an interpretation is the relatively modest levels of previous cannabis use $(1 \leq$ previous use $\leq 60$ times, $10.4 \pm$ 14.4 times on average). Nevertheless, sensitization (if it is present) to such low levels of exposure is not surprising as preclinical evidence suggests that even a single exposure to psychostimulant drugs can be sufficient to induce longlasting behavioral and cellular sensitization [66]. One may therefore speculate that the glutamatergic system may have a different steady-state homeostasis as a function of previous exposure, from where the system is particularly susceptible to destabilizing influences that may affect it, such as the acute administration of $\Delta 9$-THC. As lower baseline Glx levels and related higher $\Delta 9$-THC-induced increase were evident in individuals who developed psychotomimetic symptoms under $\Delta 9$-THC compared to individuals who did not, our findings suggest that $\Delta 9$-THC-induced psychotomimetic symptoms could be explained by $\Delta 9$-THC effects on tonic (basal) versus phasic (burst) glutamate system function, which in turn are modulated by previous cannabis use.

ACC and hippocampal Glx were not significantly increased by acute $\Delta 9$-THC administration, suggesting that glutamate alteration in the striatum may represent a specific locus of abnormality underlying sensitivity to the acute and transient psychotomimetic effects of $\Delta 9$-THC. However, this needs to be confirmed in larger samples.

Glutamate steady-state homeostasis and sensitization may also account for the apparent discrepancy between the reduction in glutamate observed in studies of chronic cannabis use in man [32-35, 37] and the increase in glutamate observed in preclinical acute challenge studies [28-31] as well as in the present acute challenge study in humans. Animal studies of chronic psychostimulant use clearly indicate drug-induced changes in glutamate regulation, such that basal glutamate levels are decreased while glutamate release is enhanced during drug exposure [64, 65]. Cannabis use may involve progressive neurochemical adaptations in glutamate function, which need to be further investigated. Determining the regional changes in glutamate function that may result 
from repeated cannabis exposure is also imperative to understanding their relevance to the acute and chronic psychoactive effects of cannabis use. To date, there is robust evidence for altered glutamate steady-state homeostasis in animal models of addiction [64, 65]. However, aberrant glutamate function has also been suggested in psychosis and related disorders. In particular, a systematic review of 63 studies investigating metabolite biomarkers of schizophrenia has indicated glutamate increase as one of the most consistent potential metabolite signatures of the disorder [67]. Increased striatal glutamate levels have also been described in subjects at ultra-high risk for psychosis, and they are also associated with conversion to psychosis [68, 69].

The major strength of this study is its design. Study subjects were recruited if they had a minimal history of cannabis use, had been abstinent from cannabis for at least 6 months, and had negligible use of other substances (alcohol, tobacco, and other illicit drugs). Therefore, we can reasonably rule out the possibility that some of the results observed could be attributed to the effects of other substance use or cannabis withdrawal, dependence, or intoxication. Moreover, for each study participant, there was an interval of at least 14 days between the two study visits. This helps exclude the possibility of any carryover effects as $\Delta$ 9-THC has been shown to have an elimination half-life of $18 \mathrm{~h}$ to 4.3 days [70]. Also, all the participants' urine samples collected at each study visit at baseline were negative for the presence of $\Delta 9$-THC. However, these strict inclusion criteria, while offering advantages in terms of a controlled sample, may at the same time limit the generalizability of the present results to the wider population of cannabis users. Also, the intravenous route for $\Delta 9-\mathrm{THC}$ administration allowed much more consistent $\Delta 9$-THC blood levels across study subjects [14], but might have similarly affected the generalizability of the results to the effects of recreational cannabis use. Another limitation of the present study is that, due to its design, it was not possible to examine the test-retest reliability of the MRS Glx measure for the regions investigated. However, evidence indicates that GM Glx in healthy subjects has relatively high reproducibility and test-retest reliability at 3 Tesla [71]. Furthermore, the within-subject design helped avoid the confounding effect of between-subject differences in the outcome variable [71].

It is worth noting that $1 \mathrm{H}-\mathrm{MRS}$ does not allow us to disentangle whether measured glutamate is from the neurotransmitter or the metabolic pool. Nevertheless, research evidence indicates that majority of the brain glutamate is cycled through the neurotransmitter pool [53] and 1H-MRS-related glutamate measures are likely to be related to glutamatergic neurotransmission [54].

It is worth considering a few other potential alternative explanations for the results presented here, such as effect of spectral quality differences, effect of $\mathrm{T} 2$ relaxation and testretest effect. Cramér-Rao lower-bound values were considerably below the $20 \%$ threshold under both drug conditions. Although full width at half maximum (FWHM) values showed a trend toward difference between the two drug conditions, they were also within the spectral quality recommended by Kreis (FWHM of metabolites $<0.07-0.1$ ppm) [72]. Collectively, they suggest good quality data, and are more informative than a comparison of quality measures across the drug conditions ( $\Delta 9-\mathrm{THC}$, placebo) [72]. Results presented here also point toward regional specificity of the acute effects of THC, as no effects on any metabolite were observed in the hippocampus and anterior cingulate, arguing against these effects being a result of spectral quality differences.

With reference to the possibility that a $\mathrm{T} 2$ relaxation effect might have occurred, decreasing the signal, this is expected to happen when a longer echo time (TE) is used [73]. Instead, the combination of a short $\mathrm{TE}$ and a long repetition time (TR) used in this study is considered to allow the acquisition of signals with minimal signal loss due to T2- and T1-weighting [73]. Nevertheless, the T2 relaxation of water, which differs between white matter, grey matter, and CSF may be different between individuals because of the presence of different fractions of these components in the MRS voxel, and arguably may introduce a systematic bias in metabolite quantification due to group difference in T2 relaxation. However, this is unlikely to have systematically affected the results of the present study as we employed a within-subject repeated measures design, thereby mitigating the effect of group difference in the fraction of grey matter, white matter, and CSF in the MRS voxel contributing to difference in $\mathrm{T} 2$ relaxation under the two drug conditions ( $\Delta 9-\mathrm{THC}$ versus Placebo). Furthermore, the randomized crossover design with counterbalanced order of drug administration employed in this study also helped mitigate a possible test-retest effect.

In summary, this study suggests that striatal glutamate levels are increased following a single dose of $\triangle 9$-THC in healthy individuals. This $\Delta 9$-THC-induced glutamate increase likely underlies the acute cannabis-induced psychotomimetic effects, as it seems to be specific to subjects experiencing psychotomimetic effects. These results also suggest that lower baseline levels of striatal glutamate may be a marker of sensitivity to the acute psychotomimetic effects of cannabis and potential sensitization to the modulation of striatal glutamate levels by $\triangle 9-T H C$ as a function of previous cannabis exposure may develop early. Collectively, these results provide novel insight into the neurochemical underpinnings of the effects of cannabis in man and may point towards potential approaches towards mitigating the adverse effects of cannabis, which may have important public health implications. 
Acknowledgements The authors acknowledge infrastructure support from the NIHR Mental Health Biomedical Research Centre at South London and Maudsley NHS Foundation Trust and King's College London. The views expressed are those of the author(s) and not necessarily those of the NHS, the NIHR or the Department of Health. Sagnik Bhattacharyya has been supported by a NIHR Clinician Scientist Award (NIHR CS-11-001) and the MRC (MR/J012149/1). Lukas Van Oudenhove has been supported by a KU Leuven Special Research Fund (BOF, Bijzonder Onderzoeksfonds). Nathalie Weltens has been supported by a KU Leuven BOF Post-Doctoral Mandate. The research leading to these results received funding from the People Programme (Marie Curie Actions) of the European Union's Seventh Framework Programme (FP7/2007-2013) under REA grant agreement no. 608765 granted to Dr. Weltens, Prof. Van Oudenhove, Dr. Bhattacharyya, and Prof. Steve Williams. The funders had no further role in study design; in the collection, analysis and interpretation of data; in the writing of the report; and in the decision to submit the paper for publication.

\section{Compliance with ethical standards}

Conflict of interest: The authors declare that they have no conflict of interest.

Ethics approval: The study was approved by the Joint South London and Maudsley (SLaM) and Institute of Psychiatry, Psychology \& Neuroscience (IoPPN) National Health Service Research Ethics Committee (PNM/13/14-38), and the investigators had a license to use $\Delta 9$-THC for research purposes.

Publisher's note: Springer Nature remains neutral with regard to jurisdictional claims in published maps and institutional affiliations.

Open Access This article is licensed under a Creative Commons Attribution 4.0 International License, which permits use, sharing, adaptation, distribution and reproduction in any medium or format, as long as you give appropriate credit to the original author(s) and the source, provide a link to the Creative Commons license, and indicate if changes were made. The images or other third party material in this article are included in the article's Creative Commons license, unless indicated otherwise in a credit line to the material. If material is not included in the article's Creative Commons license and your intended use is not permitted by statutory regulation or exceeds the permitted use, you will need to obtain permission directly from the copyright holder. To view a copy of this license, visit http://creativecommons. org/licenses/by/4.0/.

\section{References}

1. National Academies of Sciences, Engineering, and Medicine. The health effects of cannabis and cannabinoids: the current state of evidence and recommendations for research. Washington, DC: The National Academies Press; 2017.

2. Degenhardt L, Ferrari AJ, Calabria B, Hall WD, Norman RE, McGrath J, et al. The global epidemiology and contribution of cannabis use and dependence to the global burden of disease: results from the GBD 2010 study. PLoS One. 2013;8:e76635.

3. Henquet C, Krabbendam L, Spauwen J, Kaplan C, Lieb R, Wittchen HU, et al. Prospective cohort study of cannabis use, predisposition for psychosis, and psychotic symptoms in young people. BMJ. 2005;330:11.

4. Skinner R, Conlon L, Gibbons D, McDonald C. Cannabis use and non-clinical dimensions of psychosis in university students presenting to primary care. Acta Psychiatr Scand. 2011;123:21-7.
5. van Gastel WA, Wigman JT, Monshouwer K, Kahn RS, van Os J, Boks MP, et al. Cannabis use and subclinical positive psychotic experiences in early adolescence: findings from a Dutch survey. Addiction. 2012;107:381-7.

6. Moore TH, Zammit S, Lingford-Hughes A, Barnes TR, Jones $\mathrm{PB}$, Burke $\mathrm{M}$, et al. Cannabis use and risk of psychotic or affective mental health outcomes: a systematic review. Lancet. 2007;370:319-28.

7. Ouellet-Plamondon C, Abdel-Baki A, Salvat E, Potvin S. Specific impact of stimulant, alcohol and cannabis use disorders on firstepisode psychosis: 2-year functional and symptomatic outcomes. Psychol Med. 2017;47:2461-71.

8. Seddon JL, Birchwood M, Copello A, Everard L, Jones PB, Fowler D, et al. Cannabis use is associated with increased psychotic symptoms and poorer psychosocial functioning in firstepisode psychosis: a report from the UK National EDEN Study. Schizophr Bull. 2016;42:619-25.

9. Schoeler T, Monk A, Sami MB, Klamerus E, Foglia E, Brown R, et al. Continued versus discontinued cannabis use in patients with psychosis: a systematic review and meta-analysis. Lancet Psychiatry. 2016;3:215-25.

10. Patel R, Wilson R, Jackson R, Ball M, Shetty H, Broadbent M, et al. Association of cannabis use with hospital admission and antipsychotic treatment failure in first episode psychosis: an observational study. BMJ Open. 2016;6:e009888.

11. Schoeler T, Petros N, Di Forti M, Klamerus E, Foglia E, Ajnakina $\mathrm{O}$, et al. Effects of continuation, frequency, and type of cannabis use on relapse in the first 2 years after onset of psychosis: an observational study. Lancet Psychiatry. 2016;3:947-53.

12. Colizzi M, Carra E, Fraietta S, Lally J, Quattrone D, Bonaccorso $\mathrm{S}$, et al. Substance use, medication adherence and outcome one year following a first episode of psychosis. Schizophr Res. 2016;170:311-7.

13. Schoeler T, Petros N, Di Forti M, Pingault JB, Klamerus E, Foglia $\mathrm{E}$, et al. Association between continued cannabis use and risk of relapse in first-episode psychosis: a quasi-experimental investigation within an observational study. JAMA Psychiatry. 2016;73:1173-9.

14. D'Souza DC, Perry E, MacDougall L, Ammerman Y, Cooper T, $\mathrm{Wu}$ YT, et al. The psychotomimetic effects of intravenous delta-9tetrahydrocannabinol in healthy individuals: implications for psychosis. Neuropsychopharmacology. 2004;29:1558-72.

15. Bhattacharyya S, Fusar-Poli P, Borgwardt S, Martin-Santos R, Nosarti C, O'Carroll C, et al. Modulation of mediotemporal and ventrostriatal function in humans by delta9-tetrahydrocannabinol: a neural basis for the effects of Cannabis sativa on learning and psychosis. Arch Gen Psychiatry. 2009;66:442-51.

16. Bhattacharyya S, Atakan Z, Martin-Santos R, Crippa JA, Kambeitz J, Prata D, et al. Preliminary report of biological basis of sensitivity to the effects of cannabis on psychosis: AKT1 and DAT1 genotype modulates the effects of delta-9-tetrahydrocannabinol on midbrain and striatal function. Mol Psychiatry. 2012;17:1152-5.

17. Pertwee RG. The diverse CB1 and CB2 receptor pharmacology of three plant cannabinoids: delta9-tetrahydrocannabinol, cannabidiol and delta9-tetrahydrocannabivarin. $\mathrm{Br} \mathrm{J}$ Pharmacol. 2008;153:199-215.

18. Hirvonen J, Goodwin RS, Li CT, Terry GE, Zoghbi SS, Morse C, et al. Reversible and regionally selective downregulation of brain cannabinoid CB1 receptors in chronic daily cannabis smokers. Mol Psychiatry. 2012;17:642-9.

19. Lupica CR, Riegel AC, Hoffman AF. Marijuana and cannabinoid regulation of brain reward circuits. $\mathrm{Br} \mathrm{J}$ Pharmacol. 2004; 143:227-34.

20. Bossong MG, van Berckel BN, Boellaard R, Zuurman L, Schuit $\mathrm{RC}$, Windhorst $\mathrm{AD}$, et al. Delta 9-tetrahydrocannabinol induces 
dopamine release in the human striatum. Neuropsychopharmacology. 2009;34:759-66.

21. Voruganti LN, Slomka P, Zabel P, Mattar A, Awad AG. Cannabis induced dopamine release: an in-vivo SPECT study. Psychiatry Res. 2001;107:173-7.

22. Bossong MG, Mehta MA, van Berckel BN, Howes OD, Kahn RS, Stokes PR. Further human evidence for striatal dopamine release induced by administration of $\Delta 9$-tetrahydrocannabinol (THC): selectivity to limbic striatum. Psychopharmacology. 2015;232:2723-9.

23. Barkus E, Morrison PD, Vuletic D, Dickson JC, Ell PJ, Pilowsky LS, et al. Does intravenous $\Delta 9$-tetrahydrocannabinol increase dopamine release? A SPET study. J Psychopharmacol. 2011;25:1462-8.

24. Stokes PR, Mehta MA, Curran HV, Breen G, Grasby PM. Can recreational doses of THC produce significant dopamine release in the human striatum? NeuroImage. 2009;48:186-90.

25. Sami MB, Rabiner EA, Bhattacharyya S. Does cannabis affect dopaminergic signaling in the human brain? A systematic review of evidence to date. Eur Neuropsychopharmacol. 2015;25:1201-24.

26. van de Giessen E, Weinstein JJ, Cassidy CM, Haney M, Dong Z, Ghazzaoui R, et al. Deficits in striatal dopamine release in cannabis dependence. Mol Psychiatry. 2017;22:68-75.

27. Bhattacharyya S, Crippa JA, Allen P, Martin-Santos R, Borgwardt $\mathrm{S}$, Fusar-Poli $\mathrm{P}$, et al. Induction of psychosis by delta9tetrahydrocannabinol reflects modulation of prefrontal and striatal function during attentional salience processing. Arch Gen Psychiatry. 2012;69:27-36.

28. Pistis M, Ferraro L, Pira L, Flore G, Tanganelli S, Gessa GL, et al. Delta(9)-tetrahydrocannabinol decreases extracellular GABA and increases extracellular glutamate and dopamine levels in the rat prefrontal cortex: an in vivo microdialysis study. Brain Res. 2002;948:155-8.

29. Fan N, Yang H, Zhang J, Chen C. Reduced expression of glutamate receptors and phosphorylation of CREB are responsible for in vivo delta9-THC exposure-impaired hippocampal synaptic plasticity. J Neurochem. 2010;112:691-702.

30. Galanopoulos A, Polissidis A, Papadopoulou-Daifoti Z, Nomikos GG, Antoniou K. Delta(9)-THC and WIN55,212-2 affect brain tissue levels of excitatory amino acids in a phenotype-, compound-, dose-, and region-specific manner. Behav Brain Res. 2011;224:65-72.

31. Colizzi M, McGuire P, Pertwee RG, Bhattacharyya S. Effect of cannabis on glutamate signalling in the brain: a systematic review of human and animal evidence. Neurosci Biobehav Rev. 2016;64:359-81.

32. Chang L, Cloak C, Yakupov R, Ernst T. Combined and independent effects of chronic marijuana use and HIV on brain metabolites. J NeuroImmune Pharmacol. 2006;1:65-76.

33. Muetzel RL, Marjanska M, Collins PF, Becker MP, Valabregue $\mathrm{R}$, Auerbach EJ, et al. In vivo ${ }^{1} \mathrm{H}$ magnetic resonance spectroscopy in young-adult daily marijuana users. NeuroImage Clin. 2013;2:581-9.

34. Prescot AP, Renshaw PF, Yurgelun-Todd DA. $\gamma$-Amino butyric acid and glutamate abnormalities in adolescent chronic marijuana smokers. Drug Alcohol Depend. 2013;129:232-9.

35. Prescot AP, Locatelli AE, Renshaw PF, Yurgelun-Todd DA. Neurochemical alterations in adolescent chronic marijuana smokers: a proton MRS study. NeuroImage. 2011;57:69-75.

36. Sung YH, Carey PD, Stein DJ, Ferrett HL, Spottiswoode BS, Renshaw PF, et al. Decreased frontal N-acetylaspartate levels in adolescents concurrently using both methamphetamine and marijuana. Behav Brain Res. 2013;246:154-61.

37. Bernier D, Bartha R, McAllindon D, Hanstock CC, Marchand Y, Dillen $\mathrm{KNH}$, et al. Illness versus substance use effects on the frontal white matter in early phase schizophrenia: a 4 Tesla (1)HMRS study. Schizophr Res. 2016;175:4-11.
38. D'Souza DC, Pittman B, Perry E, Simen A. Preliminary evidence of cannabinoid effects on brain-derived neurotrophic factor (BDNF) levels in humans. Psychopharmacology. 2009;202:569-78.

39. Bhattacharyya S, Morrison PD, Fusar-Poli P, Martin-Santos R, Borgwardt S, Winton-Brown T, et al. Opposite effects of delta9-tetrahydrocannabinol and cannabidiol on human brain function and psychopathology. Neuropsychopharmacology. 2010;35:764-74.

40. Kay SR, Fiszbein A, Opler LA. The positive and negative syndrome scale (PANSS) for schizophrenia. Schizophr Bull. 1987;13:261-76.

41. Guillen-Riquelme A, Buela-Casal G. Meta-analysis of group comparison and meta-analysis of reliability generalization of the State-Trait Anxiety Inventory Questionnaire (STAI). Rev Esp Salud Publica. 2014;88:101-12.

42. Folstein MF, Luria R. Reliability, validity, and clinical application of the Visual Analogue Mood Scale. Psychol Med. 1973;3:479-86.

43. Mathew RJ, Wilson WH, Humphreys DF, Lowe JV, Wiethe KE. Regional cerebral blood flow after marijuana smoking. J Cereb Blood Flow Metab. 1992;12:750-8.

44. Stone JM. Imaging the glutamate system in humans: relevance to drug discovery for schizophrenia. Curr Pharm Des. 2009;15: 2594-602.

45. Stone JM, Day F, Tsagaraki H, Valli I, McLean MA, Lythgoe DJ, et al. Glutamate dysfunction in people with prodromal symptoms of psychosis: relationship to gray matter volume. Biol Psychiatry. 2009;66:533-9.

46. Stone JM, Dietrich C, Edden R, Mehta MA, De Simoni S, Reed LJ, et al. Ketamine effects on brain GABA and glutamate levels with 1H-MRS: relationship to ketamine-induced psychopathology. Mol Psychiatry. 2012;17:664-5.

47. Valli I, Stone J, Mechelli A, Bhattacharyya S, Raffin M, Allen P, et al. Altered medial temporal activation related to local glutamate levels in subjects with prodromal signs of psychosis. Biol Psychiatry. 2011;69:97-9.

48. Provencher SW. Estimation of metabolite concentrations from localized in vivo proton NMR spectra. Magn Reson Med. 1993;30:672-9.

49. Fulford D, Pearson R, Stuart BK, Fisher M, Mathalon DH, Vinogradov $\mathrm{S}$, et al. Symptom assessment in early psychosis: the use of well-established rating scales in clinical high-risk and recent-onset populations. Psychiatry Res. 2014;220:1077-83.

50. Bhattacharyya S, Sainsbury T, Allen P, Nosarti C, Atakan Z, Giampietro V, et al. Increased hippocampal engagement during learning as a marker of sensitivity to psychotomimetic effects of delta-9-THC. Psychol Med. 2018;48:2748-56.

51. Kaiser LG, Schuff N, Cashdollar N, Weiner MW. Age-related glutamate and glutamine concentration changes in normal human brain: $1 \mathrm{H}$ MR spectroscopy study at $4 \mathrm{~T}$. Neurobiol Aging. 2005;26:665-72.

52. Newsholme P, Procopio J, Lima MM, Pithon-Curi TC, Curi R. Glutamine and glutamate- their central role in cell metabolism and function. Cell Biochem Funct. 2003;21:1-9.

53. Rothman DL, Behar KL, Hyder F, Shulman RG. In vivo NMR studies of the glutamate neurotransmitter flux and neuroenergetics: implications for brain function. Annu Rev Physiol. 2003;65:401-27.

54. Yuksel C, Ongur D. Magnetic resonance spectroscopy studies of glutamate-related abnormalities in mood disorders. Biol Psychiatry. 2010;68:785-94.

55. Somogyi P, Bolam JP, Smith AD. Monosynaptic cortical input and local axon collaterals of identified striatonigral neurons. A light and electron microscopic study using the Golgi-peroxidase transportdegeneration procedure. J Comp Neurol. 1981;195:567-84. 
56. Sadikot AF, Parent A, Smith Y, Bolam JP. Efferent connections of the centromedian and parafascicular thalamic nuclei in the squirrel monkey: a light and electron microscopic study of the thalamostriatal projection in relation to striatal heterogeneity. J Comp Neurol. 1992;320:228-42.

57. Cooper DC. The significance of action potential bursting in the brain reward circuit. Neurochem Int. 2002;41:333-40.

58. Tepper JM, Martin LP, Anderson DR. GABAA receptor-mediated inhibition of rat substantia nigra dopaminergic neurons by pars reticulata projection neurons. J Neurosci. 1995;15:3092-103.

59. Melis M, Pistis M, Perra S, Muntoni AL, Pillolla G, Gessa GL. Endocannabinoids mediate presynaptic inhibition of glutamatergic transmission in rat ventral tegmental area dopamine neurons through activation of CB1 receptors. J Neurosci. 2004;24:53-62.

60. Szabo B, Muller T, Koch H. Effects of cannabinoids on dopamine release in the corpus striatum and the nucleus accumbens in vitro. J Neurochem. 1999;73:1084-9.

61. Cheer JF, Marsden CA, Kendall DA, Mason R. Lack of response suppression follows repeated ventral tegmental cannabinoid administration: an in vitro electrophysiological study. Neuroscience. 2000;99:661-7.

62. Matsuda LA, Bonner TI, Lolait SJ. Localization of cannabinoid receptor mRNA in rat brain. J Comp Neurol. 1993;327:535-50.

63. Reynolds SM, Zahm DS. Specificity in the projections of prefrontal and insular cortex to ventral striatopallidum and the extended amygdala. J Neurosci. 2005;25:11757-67.

64. Parsegian A, See RE. Dysregulation of dopamine and glutamate release in the prefrontal cortex and nucleus accumbens following methamphetamine self-administration and during reinstatement in rats. Neuropsychopharmacology. 2014;39:811-22.
65. Gabriele A, Pacchioni AM, See RE. Dopamine and glutamate release in the dorsolateral caudate putamen following withdrawal from cocaine self-administration in rats. Pharmacol Biochem Behav. 2012;103:373-9.

66. Wolf ME, Sun X, Mangiavacchi S, Chao SZ. Psychomotor stimulants and neuronal plasticity. Neuropharmacology. 2004;47:61-79.

67. Davison J, O'Gorman A, Brennan L, Cotter DR. A systematic review of metabolite biomarkers of schizophrenia. Schizophr Res. 2018;195:32-50.

68. de la Fuente-Sandoval C, Leon-Ortiz P, Azcarraga M, Favila R, Stephano S, Graff-Guerrero A. Striatal glutamate and the conversion to psychosis: a prospective $1 \mathrm{H}-\mathrm{MRS}$ imaging study. Int $\mathrm{J}$ Neuropsychopharmacol. 2013;16:471-5.

69. de la Fuente-Sandoval C, Reyes-Madrigal F, Mao X, Leon-Ortiz P, Rodriguez-Mayoral O, Solis-Vivanco $\mathrm{R}$, et al. Cortico-striatal GABAergic and glutamatergic dysregulations in subjects at ultrahigh risk for psychosis investigated with proton magnetic resonance spectroscopy. Int J Neuropsychopharmacol. 2015;19:pyv105.

70. Kelly P, Jones RT. Metabolism of tetrahydrocannabinol in frequent and infrequent marijuana users. J Anal Toxicol. 1992;16:228-35.

71. Gasparovic C, Bedrick EJ, Mayer AR, Yeo RA, Chen H, Damaraju E, et al. Test-retest reliability and reproducibility of short-echo-time spectroscopic imaging of human brain at $3 \mathrm{~T}$. Magn Reson Med. 2011;66:324-32.

72. Kreis R. Issues of spectral quality in clinical $1 \mathrm{H}$-magnetic resonance spectroscopy and a gallery of artifacts. NMR Biomed. 2004;17:361-81.

73. van der Graaf M. In vivo magnetic resonance spectroscopy: basic methodology and clinical applications. Eur Biophys J. 2010;39:527-40. 